\title{
Robotic nipple-sparing mastectomy complication rate compared to traditional nipple-sparing mastectomy: a systematic review and meta-analysis
}

\author{
M. D. Filipe ${ }^{1}$ E. de Bock ${ }^{1}$ - E. L. Postma ${ }^{2}$ O. W. Bastian ${ }^{1}$ - P. P. A. Schellekens ${ }^{3} \cdot$ M. R. Vriens ${ }^{1} \cdot$ A. J. Witkamp $^{1}$. \\ M. C. Richir ${ }^{1}$
}

Received: 4 April 2021 / Accepted: 9 June 2021 / Published online: 14 June 2021

(c) The Author(s) 2021

\begin{abstract}
Breast cancer is worldwide the most common cause of cancer in women and causes the second most common cancer-related death. Nipple-sparing mastectomy (NSM) is commonly used in therapeutic and prophylactic settings. Furthermore, (preventive) mastectomies are, besides complications, also associated with psychological and cosmetic consequences. Robotic NSM (RNSM) allows for better visualization of the planes and reducing the invasiveness. The aim of this study was to compare the postoperative complication rate of RNSM to NSM. A systematic search was performed on all (R)NSM articles. The primary outcome was determining the overall postoperative complication rate of traditional NSM and RNSM. Secondary outcomes were comparing the specific postoperative complication rates: implant loss, hematoma, (flap)necrosis, infection, and seroma. Forty-nine studies containing 13,886 cases of (R)NSM were included. No statistically significant differences were found regarding postoperative complications (RNSM 3.9\%, NSM 7.0\%, $p=0.070$ ), postoperative implant loss (RNSM 4.1\%, NSM 3.2\%, $p=0.523$ ), hematomas (RNSM 4.3\%, NSM 2.0\%, $p=0.059)$, necrosis (RNSM 4.3\%, NSM 7.4\%, $p=0.230)$, infection (RNSM 8.3\%, NSM 4.0\%, $p=0.054$ ) or seromas (RNSM 3.0\%, NSM 2.0\%, $p=0.421$ ). Overall, there are no statistically significant differences in complication rates between NSM and RNSM.
\end{abstract}

Keywords Robot assisted nipple sparing mastectomy $\cdot$ Surgery $\cdot$ Minimally invassive

\section{Introduction}

Breast cancer is the most common type of cancer in women and the second most common cause of death due to cancer in women worldwide [1]. There are approximately 17,000 new cases of breast cancer in the Netherlands every year. In addition, over 3,000 women of the Dutch population annually die

M. D. Filipe

m.d.filipe@umcutrecht.nl

M. C. Richir

m.c.richir@umcutrecht.nl

1 Department of Surgery, Cancer Centre, University Medical Centre Utrecht, PO Box 85500, 3508 GA Utrecht, The Netherlands

2 Department of Surgery, St. Antonius Hospital, Nieuwegein, The Netherlands

3 Department of Plastic Surgery, University Medical Centre Utrecht, Utrecht, The Netherlands due to breast cancer [2]. Hereditary breast cancer accounts for up to 5-10\% of all breast cancers. Two high-penetrance genes (BRCA1 and BRCA2) are responsible for about $16 \%$ of the familial risk of breast cancers and associated with a $60-80 \%$ lifetime risk of developing breast cancer $[3,4]$.

Surgical resection of the primary tumor is the treatment of choice in patients with new-onset breast cancer. Tumor stage and molecular characteristics determine the type of surgery. Most patients are treated by breast-conserving surgery followed by radiation therapy (breast-conserving therapy, BCT) or mastectomy with or without breast reconstruction $[5,6]$.

Currently, the ultimate prevention in women with hereditary breast cancer is bilateral prophylactic mastectomy [7]. Consequently, this means that $20-40 \%$ of these patients undergo mastectomies without signs of malignancy. Unfortunately, a part of these patients will develop complications or experience poor cosmetic results and carry a significant psychological burden [8, 9]. 
Nipple-sparing mastectomy (NSM) was initially reserved for the prophylactic treatment of women with a high risk of developing breast cancer [10]. However, NSM has been increasingly used therapeutically for breast cancer where the nipple-areolar complex is not involved [11-14]. One of the most important challenges of nipple-sparing mastectomy is achieving adequate exposure to performing precise dissection in areas that are remote from the skin incision [15].

Robotic nipple-sparing mastectomy (RNSM) is a relatively new technique that allows for better visualization of tissue planes and exposes tissue that is challenging to reach with traditional nipple-sparing mastectomy techniques [16-18]. Previous research has not only demonstrated the feasibility and safety of RNSM but also that RNSM has a steep learning curve [16, 19]. However, there are currently no studies comparing the complication rate of RNSM to NSM. Therefore, the aim of this study is to compare postoperative complications of patients undergoing traditional NSM to RNSM followed by immediate breast reconstruction.

\section{Materials and methods}

This systematic review and meta-analysis were performed according to the guidelines of the requirements of the PRISMA Checklist for meta-analysis [20]. A systematic literature search was performed in the PubMed, Embase and Cochrane Library databases. The search strategy was performed on all index tests (NSM and RNSM) and their synonyms. The full electronic search strategy can be found in the supplementary data (Supplementary Material 1). After the removal of duplicates, two authors (MF, EB) independently screened articles by title and abstract. The two authors discussed discordant judgments until consensus was reached. The full articles were independently screened for eligibility based on predefined inclusion and exclusion criteria.

\section{Selection of studies}

Full texts were retrieved for studies that evaluated (robot) nipple-sparing mastectomy, reported original data and were written in English.

1. Participants: patients undergoing therapeutic or prophylactic (R)NSM with immediate breast reconstruction (IBR).

2. Intervention: (R)NSM.

3. Outcome: postoperative complications (implant loss, hematoma, necrosis, infection or seroma).

Studies were excluded from systematic review based on the following criteria:
1. Not possible to determine whether patients had immediate reconstruction.

2. Non-robotic endoscopic NSM and/or reconstruction.

3. Intra-operative radiotherapy.

4. Case report, review and conference abstracts.

\section{Risk of bias}

The ROBINS-I Tool was used to evaluate the quality of each eligible study [21]. The entire scale constituted seven domains for the risk of bias; confounding, selection of participants, classification of interventions, deviations from intended interventions, missing data, measurement of outcomes, and selection of the reported result. Each domain was judged for three levels of bias: low risk, intermediate/ unclear risk, or high risk of bias. Full assessment criteria can be found in the supplementary data (Supplementary Material 2).

\section{Statistical analysis}

Pairwise meta-analysis was performed to compare complication rates with $95 \%$ confidence intervals (CI) of RNSM to NSM in studies. Pooled postoperative complication rates were determined using random effects models. $p$ values under 0.05 were considered statistically significant.

All calculations were performed using RStudio 1.2.5001 (with $\mathrm{R}$ version: $\times 64$ 3.6.3). Additionally, statistical packages meta, mada, metafor, gemtc, mvmeta and were used for all computations of the meta-analyses. Visualization of plots was done using the ggplot2 package.

\section{Results}

One thousand one hundred and sixteen citations were identified by the search and, after removing duplicates, 95 potentially eligible articles were retrieved in full text (Fig. 1). Overall, 13,886 (R)NSM were performed in 49 studies with an average of 294.6 participants per study [supplementary Table 1].

Supplementary Table 1 shows the studies included in the analysis and their characteristics. Seven studies described postoperative complications of RNSM and 42 studies described the postoperative complications of NSM. In total, 13 out of 225 mastectomies (3.9\%) developed postoperative complications in RNSM while 1,056 out of 13,661 NSM (7.0\%) developed postoperative complications. This difference was not statistically significant $(p=0.070)$ (Fig. 2). 


\section{IPRISME |PRISMA 2009 Flow Diagram}
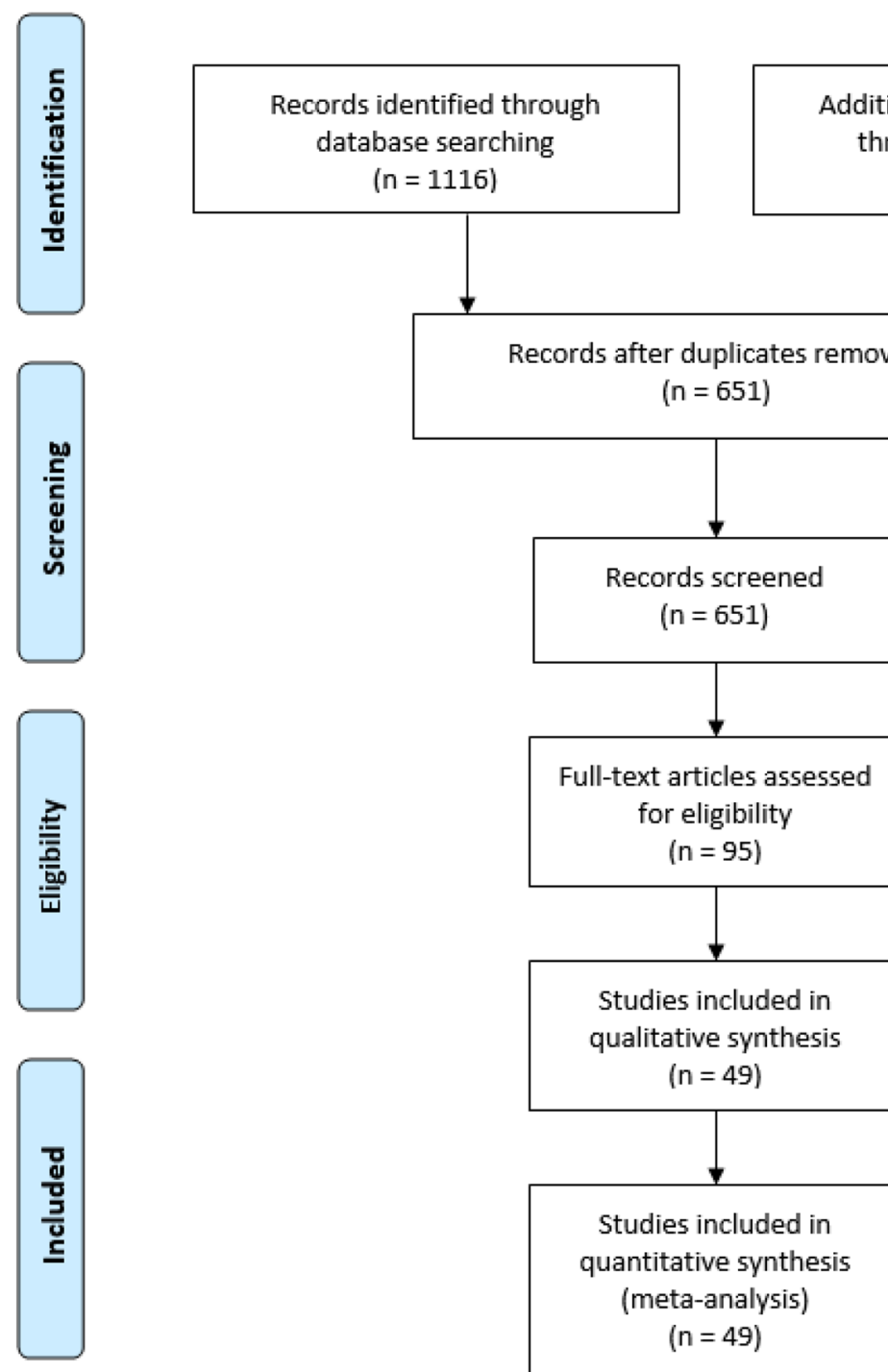

Fig. 1 Flow chart showing literature search and study selection with 49 relevant studies ultimately enrolled in this meta-analysis on the complication rate of (robotic) nipple-sparing mastectomy

\section{Different postoperative complications}

Detailed meta-analysis of the different complications of each study can be found in Supplementary Figs. 1-5. Postmastectomy implant removal occurred in $4.1 \%$ of RNSM and in $3.2 \%$ in NSM. This difference was not statistically significant $(p=0.523)$. Furthermore, post-mastectomy hematoma occurred more often in RNSM (4.3\%) than in
NSM (2.0\%) but this difference was not statistically significant $(p=0.059)$. Moreover, post-mastectomy necrosis and seroma occurred in respectively $4.3 \%$ and $3.0 \%$ in RNSM and $7.4 \%$ and $2.0 \%$ in NSM. These differences were not statistically significant. Finally, postoperative mastectomy infection occurred more often in RNSM (8.3\%) than in NSM (4.0\%) but this was not statistically significant $(p=0.054)($ Table 1$)$. 
Fig. 2 Complication rates. $(R)$ $N S M$ (robotic) nipple-sparing mastectomy, $C I$ confidence interval

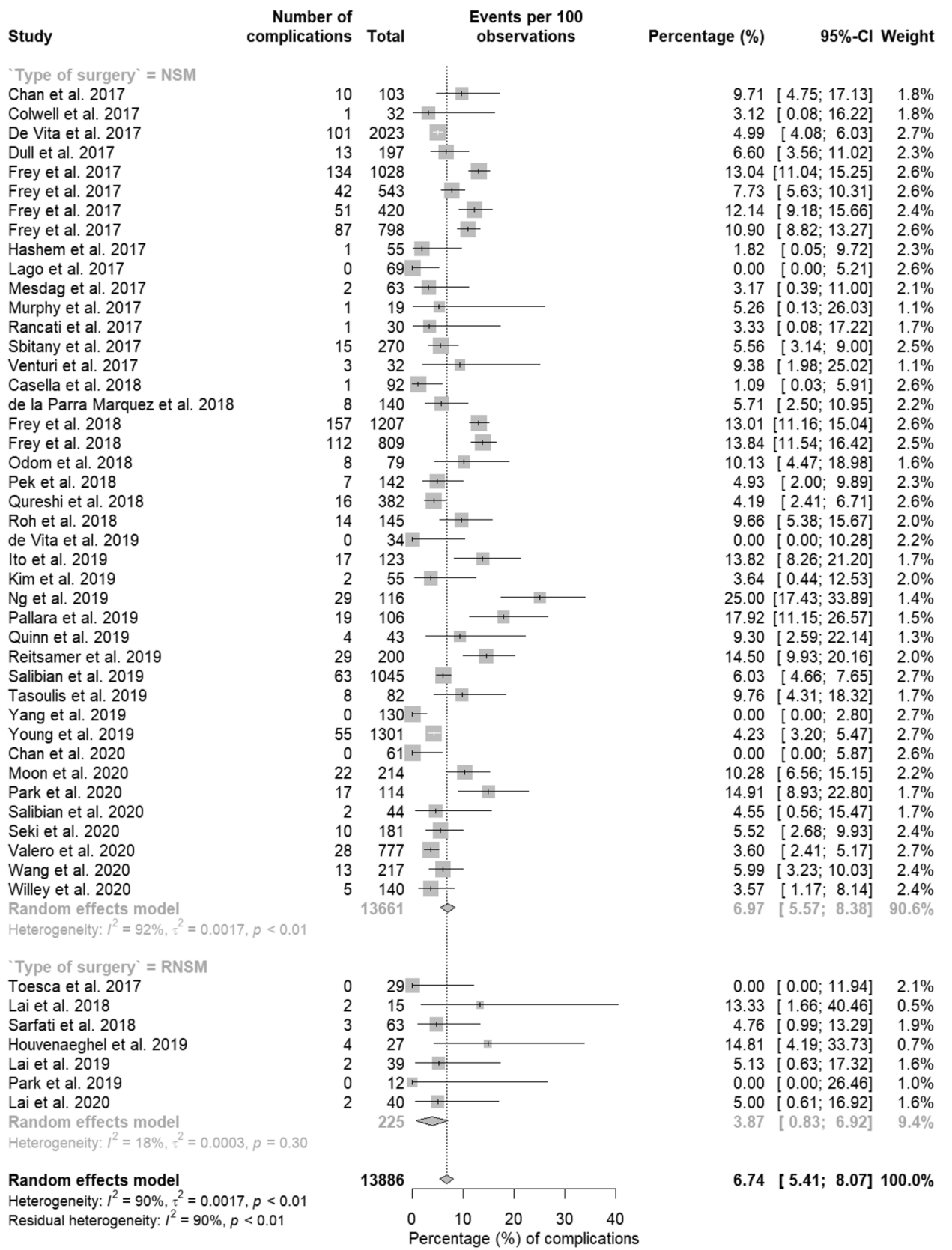

\section{Risk of bias}

The result of the ROBINS-I Tool revealed that all the included studies were of sufficient quality. This was for risks of bias domains and applicability domains (Fig. 3). Risk assessment of every study can be found in Supplementary Material 4.
Table 1 Pooled complication rates of (R)NSM

\begin{tabular}{llll}
\hline Parameter, \% (95\% CI) & RNSM (\%) & NSM (\%) & $p$ value \\
\hline Total complications & $3.9(0.8-6.9)$ & $7.0(5.6-8.4)$ & 0.070 \\
Implant loss & $4.1(1.9-8.7)$ & $3.2(2.4-4.2)$ & 0.523 \\
Hematoma & $4.3(2.0-9.1)$ & $2.0(1.7-2.4)$ & 0.059 \\
Necrosis & $4.3(1.8-10.0)$ & $7.4(5.8-9.3)$ & 0.230 \\
Infection & $8.3(4.2-15.8)$ & $4.0(3.0-5.3)$ & 0.054 \\
Seroma & $3.0(1.3-7.1)$ & $2.0(1.3-3.1)$ & 0.421 \\
\hline
\end{tabular}

(R)NSM (robotic) nipple-sparing mastectomy, CI confidence interval 

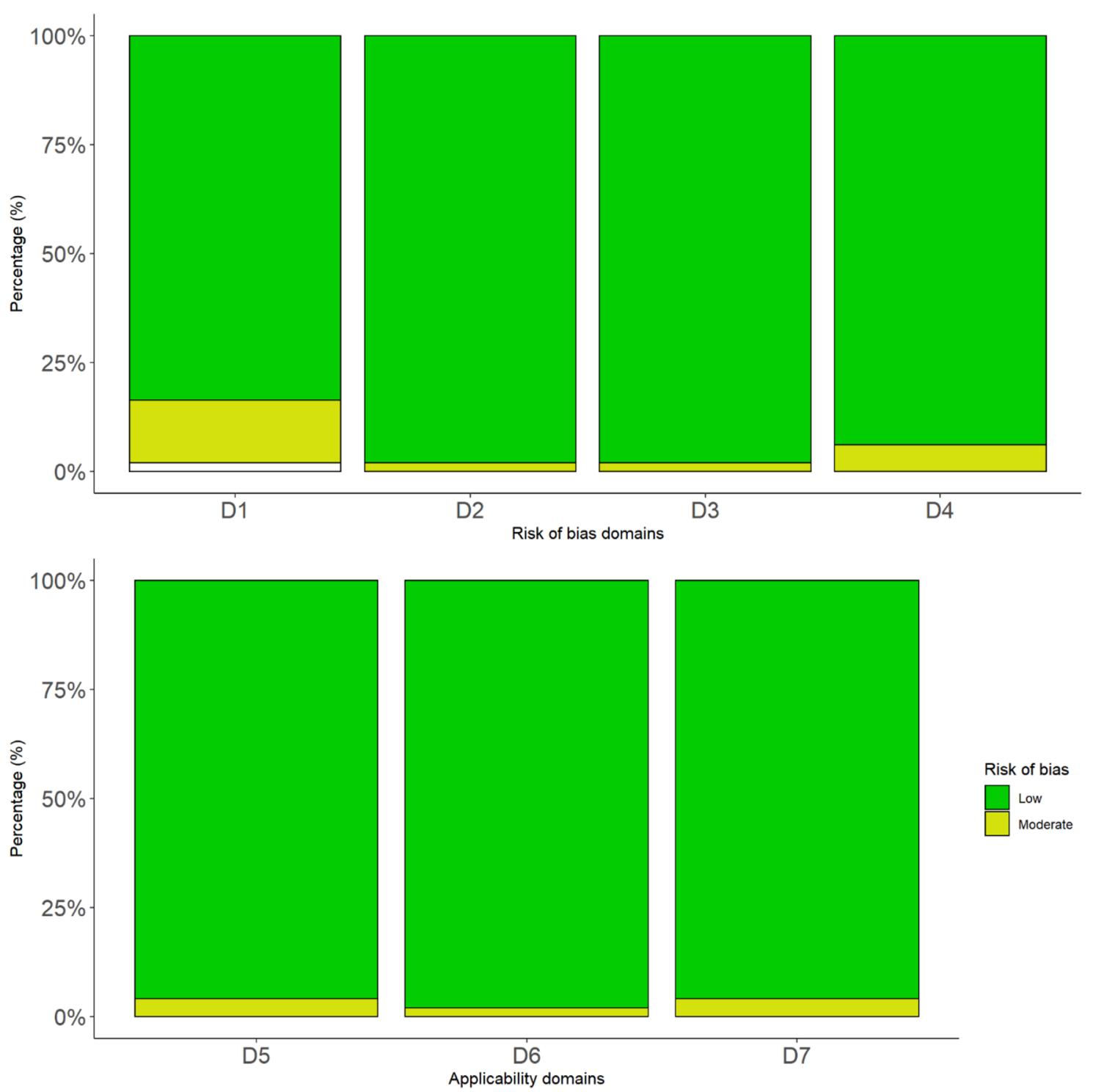

Fig. 3 Summary of the risks of bias and applicability domains. $\mathrm{D} 1=$ Bias due to confounding D2=Bias in selection of participants into the study; D3=Bias in the classification of interventions;

\section{Discussion}

This meta-analysis, including 49 studies, is the first study to compare the complication rate of RNSM to NSM in patients undergoing prophylactic or therapeutic mastectomy. The current study shows that patients undergoing RNSM do not have an increased risk of developing postoperative complications when compared to NSM.

Approximately $40 \%$ of patients with invasive breast cancer and $30 \%$ of patients with ductal carcinoma in situ (DCIS) undergo mastectomy [22]. Additionally, there are many BRCA1 or BRCA2 gene mutation carriers that undergo prophylactic mastectomies. As mastectomies are
D4 $=$ Bias due to deviations from intended interventions; D5 = Bias due to missing data; D6=Bias in measurements of outcomes; D7 $=$ Bias in the selection of the reported result

accompanied by complications along with serious cosmetic and psychological consequences [8,9]. For these patients, it is essential to keep the surgical intervention as minimally invasive as possible, while preserving the (oncological) safety. RNSM has shown to be feasible and safe, both as a therapeutic and a prophylactic treatment $[19,23]$. To obtain information to provide a reliable insight into the postoperative complications between NSM and RNSM procedures, a literature search was performed. The overall complication rate was 3.9\% after RNSM and $7.0 \%$ after NSM. This difference was not statistically different. Considering individual complications, patients did not appear to have an increased risk of postoperative 
implant loss or developing hematomas, infections, seromas or necrosis due to RNSM.

While there are no differences in complications between traditional NSM and RNSM, RNSM does offer certain advantages. Robotic surgery in general provides smaller incisions compared to open surgery [24]. Furthermore, RNSM allows for better visualization of the planes and exposes tissue that is challenging to reach with traditional nipple-sparing mastectomy techniques [16-18]. Another advantage of the scopic nature of the RNSM could be that this technique allows enhanced imaging techniques that could detect (pre)cancerous breast cancer lesions. One of these techniques is narrow-band imaging (NBI). NBI is a well-established technique used during colonoscopy, cystoscopy, and bronchoscopy to detect (pre)cancerous lesions of epithelial origin [25-27]. Therefore, it is reasonable to assume that NBI might be useful since (pre) malignancy is known to show different patterns of vascularization compared to healthy breast tissue [28, 29]. Furthermore, (pre)malignant epithelial lesions show an aberrant pattern under fluorescent light by which they become detectable. This technique is already used extensively to help identify pathologies within the airways, larynx, and colon [30-32]. Moreover, studies have also shown promising results of (auto) fluorescence for the detection of (pre) cancerous lesions of the breast [33, 34]. Consequently, enhanced imaging techniques, such as NBI and (auto) fluorescence, combined with robotic surgery could improve margin determination in patients undergoing breast cancer surgery. Furthermore, another advantage of robotic surgery is that it provides better ergonomics for the surgeon when compared to traditional surgery [35]. A possible downside of robotic breast surgery, as with many other types of robotic surgery, is that the preparations (docking and positioning of the patient) and the procedure itself takes longer [16]. The longer operation time results, along with the higher material costs, in an overall more expensive procedure.

A recent systematic review also concluded that RNSM is safe to use with acceptable short-term outcomes [36]. However, the current study quantifies (by means of a meta-analysis) the risk of postoperative complications in patients undergoing RNSM and compares said risk to traditional mastectomy.

This study has some limitations. The number of patients in the studies describing RNSM are relatively low compared to traditional NSM. This can be attributed to the fact that RNSM is a relatively new technique. Furthermore, the current study did not describe which percentage of the mastectomies were prophylactic or therapeutic. This might have an effect on the risk of postoperative complications since neo-adjuvant therapy increases the risk of postoperative complications [37]. Although RNSM has great potential, additional prospective research is warranted to further determine oncological safety, long-term postoperative complications and patient-reported outcomes in patients undergoing RNSM. Currently, in most countries RNSM is off-label. Therefore, the previously proposed additional research and the results of the current study could help to make RNSM a recommended viable option for women requiring mastectomy.

In conclusion, this study shows that there is no significant difference in the postoperative complication rate of RNSM compared to traditional NSM. Therefore, RNSM can be used safely in patients that require a prophylactic or therapeutic mastectomy.

Supplementary Information The online version contains supplementary material available at https://doi.org/10.1007/s11701-021-01265-w.

Funding The authors did not receive support from any organization for the submitted work.

\section{Declarations}

Conflict of interest M.D. Filipe, E. de Bock, E.L. Postma, O.W. Bastian declares, P.P.A. Schellekens, M.R. Vriens, A.J. Witkamp and M.C. Richir declare that they have no conflict of interest. All authors certify that they have no affiliations with or involvement in any organization or entity with any financial interest or non-financial interest in the subject matter or materials discussed in this manuscript.

Informed consent This article does not contain any studies with human or animal subjects performed by any of the authors.

Open Access This article is licensed under a Creative Commons Attribution 4.0 International License, which permits use, sharing, adaptation, distribution and reproduction in any medium or format, as long as you give appropriate credit to the original author(s) and the source, provide a link to the Creative Commons licence, and indicate if changes were made. The images or other third party material in this article are included in the article's Creative Commons licence, unless indicated otherwise in a credit line to the material. If material is not included in the article's Creative Commons licence and your intended use is not permitted by statutory regulation or exceeds the permitted use, you will need to obtain permission directly from the copyright holder. To view a copy of this licence, visit http://creativecommons.org/licenses/by/4.0/.

\section{References}

1. Siegel RL, Miller KD, Jemal A (2018) Cancer statistics, 2018. CA Cancer J Clin 68(1):7-30

2. Vondeling GT, Menezes GL, Dvortsin EP, Jansman FGA, Konings IR, Postma MJ et al (2018) Burden of early, advanced and metastatic breast cancer in The Netherlands. BMC Cancer 18(1):262-263

3. Ford D, Easton DF, Stratton M, Narod S, Goldgar D, Devilee P et al (1998) Genetic heterogeneity and penetrance analysis of the 
BRCA1 and BRCA2 genes in breast cancer families. The Breast Cancer Linkage Consortium. Am J Hum Genet 62(3):676-689

4. Tung N, Battelli C, Allen B, Kaldate R, Bhatnagar S, Bowles K et al (2015) Frequency of mutations in individuals with breast cancer referred for BRCA1 and BRCA2 testing using next-generation sequencing with a 25-gene panel. Cancer 121(1):25-33

5. Hartmann-Johnsen OJ, Karesen R, Schlichting E, Nygard JF (2015) Survival is better after breast conserving therapy than mastectomy for early stage breast cancer: a registry-based follow-up study of Norwegian women primary operated between 1998 and 2008. Ann Surg Oncol 22(12):3836-3845

6. van Maaren MC, de Munck L, de Bock GH, Jobsen JJ, van Dalen $\mathrm{T}$, Linn SC et al (2016) 10 year survival after breast-conserving surgery plus radiotherapy compared with mastectomy in early breast cancer in the Netherlands: a population-based study. Lancet Oncol 17(8):1158-1170

7. Casella D, Di Taranto G, Marcasciano M, Sordi S, Kothari A, Kovacs T et al ( 2018) Nipple-sparing bilateral prophylactic mastectomy and immediate reconstruction with TiLoop ${ }^{\circledR}$ Bra mesh in BRCA1/2 mutation carriers: a prospective study of long-term and patient reported outcomes using the BREAST-Q. Breast 39:8-13

8. Kim H, Park S-J, Woo K-J, Bang SI (2019) Comparative study of nipple-areola complex position and patient satisfaction after unilateral mastectomy and immediate expander-implant reconstruction nipple-sparing mastectomy versus skin-sparing mastectomy. Aesthetic Plast Surg 43(2):313-327

9. Pek W-S, Tan B-K, Ru Ng YY, Kiak Mien Tan V, Rasheed MZ, Kiat Tee Tan B, et al (2018) Immediate breast reconstruction following nipple-sparing mastectomy in an Asian population: aesthetic outcomes and mitigating nipple-areolar complex necrosis. Arch Plast Surg 45(3):229-238

10. Newman LA, Kuerer HM, Hunt KK, Vlastos G, Ames FC, Ross MI et al (2000) Prophylactic mastectomy. J Am Coll Surg 191(3):322-330

11. Mesdag V, Régis C, Tresch E, Chauvet M-P, Boulanger L, Collinet $P$ et al (2017) Nipple sparing mastectomy for breast cancer is associated with high patient satisfaction and safe oncological outcomes. J Gynecol Obstet Hum Reprod 46(8):637-642

12. Lago V, Maisto V, Gimenez-Climent J, Vila J, Vazquez C, Estevan R (2018) Nipple-sparing mastectomy as treatment for patients with ductal carcinoma in situ: a 10-year follow-up study. Breast J 24(3):298-303

13. Chan YH-Y, Yau W-M, Cheung PS-Y (2018) Oncological safety and technical feasibility of nipple-sparing mastectomy for breast cancer: the hong kong experience. World J Surg 42(5):1375-1383

14. Galimberti V, Vicini E, Corso G, Morigi C, Fontana S, Sacchini $\mathrm{V}$ et al (2017) Nipple-sparing and skin-sparing mastectomy: review of aims, oncological safety and contraindications. Breast 34:S82-S84

15. Coopey SB, Mitchell SD (2017) Nipple-sparing mastectomy: pitfalls and challenges. Ann Surg Oncol 24(10):2863-2868

16. Toesca A, Peradze N, Manconi A, Galimberti V, Intra M, Colleoni $\mathrm{M}$ et al (2017) Robotic nipple-sparing mastectomy for the treatment of breast cancer: feasibility and safety study. Breast 31:51-56

17. Park HS, Lee J, Lee DW, Song SY, Lew DH, Kim S Il, et al. Robot-assisted Nipple-sparing Mastectomy with Immediate Breast Reconstruction: An Initial Experience. Sci Rep [Internet]. 2019;9(1):15669.

18. Lai H-W, Chen S-T, Lin S-L, Chen C-J, Lin Y-L, Pai S-H, et al (2019) Robotic nipple-sparing mastectomy and immediate breast reconstruction with gel implant: technique, preliminary results and patient-reported cosmetic outcome. Ann Surg Oncol 26(1):42-52

19. Houvenaeghel G, Bannier M, Rua S, Barrou J, Heinemann M, Van TA et al (2019) Breast cancer robotic nipple sparing mastectomy: evaluation of several surgical procedures and learning curve. World J Surg Oncol. https://doi.org/10.1186/s12957-019-1567-y

20. Liberati A, Altman DG, Tetzlaff J, Mulrow C, Gotzsche PC, Ioannidis JPA et al (2009) The PRISMA statement for reporting systematic reviews and meta-analyses of studies that evaluate healthcare interventions: explanation and elaboration. BMJ 339:b2700

21. Sterne JA, Hernán MA, Reeves BC, Savović J, Berkman ND, Viswanathan $\mathrm{M}$ et al (2016) ROBINS-I: a tool for assessing risk of bias in non-randomised studies of interventions. BMJ 355:i4919

22. van Bommel ACM, Spronk PER, Vrancken Peeters M-JTFD, Jager A, Lobbes M, Maduro JH et al (2017) Clinical auditing as an instrument for quality improvement in breast cancer care in the Netherlands: the national NABON breast cancer audit. J Surg Oncol 115(3):243-249

23. Houvenaeghel G, Cohen M, Ribeiro SR, Barrou J, Heinemann M, Frayret C et al (2020) Robotic nipple-sparing mastectomy and immediate breast reconstruction with robotic latissimus dorsi flap harvest: technique and results. Surg Innov 18:155335062091791

24. van der Sluis PC, Ruurda JP, van der Horst S, Verhage RJ, Besselink MG, Prins MJ et al (2012) Robot-assisted minimally invasive thoraco-laparoscopic esophagectomy versus open transthoracic esophagectomy for resectable esophageal cancer, a randomized controlled trial (ROBOT trial). Trials 13(1):230

25. Singh R, Mei SC, Sethi S (2011) Advanced endoscopic imaging in Barrett's oesophagus: a review on current practice. World J Gastroenterol 17(38):4271-4276

26. Jichlinski P, Lovisa B (2011) High magnification cystoscopy in the primary diagnosis of bladder tumors. Curr Opin Urol 21(5):398-402

27. Tanaka S, Sano Y (2011) Aim to unify the narrow band imaging (NBI) magnifying classification for colorectal tumors: current status in Japan from a summary of the consensus symposium in the 79th Annual Meeting of the Japan Gastroenterological Endoscopy Society. Dig Endosc 23(Suppl 1):131-139

28. Gadre SA, Perkins GH, Sahin AA, Sneige N, Deavers MT, Middleton LP (2008) Neovascularization in mucinous ductal carcinoma in situ suggests an alternative pathway for invasion. Histopathology 53(5):545-553

29. Li L, Wang K, Sun X, Wang K, Sun Y, Zhang G et al (2015) Parameters of dynamic contrast-enhanced MRI as imaging markers for angiogenesis and proliferation in human breast cancer. Med Sci Monit 21:376-382

30. Ogihara T, Watanabe H, Namihisa A, Kobayashi O, Miwa H, Sato N (1999) Clinical experience using a real time autofluorescence endoscopy system in the gastrointestinal tract. Diagn Ther Endosc 5(2):119-124

31. Ikeda N, Honda H, Hayashi A, Usuda J, Kato Y, Tsuboi M et al (2006) Early detection of bronchial lesions using newly developed videoendoscopy-based autofluorescence bronchoscopy. Lung Cancer 52(1):21-27

32. van der Heijden EH, Hoefsloot W, van Hees HW, Schuurbiers OC (2015) High definition bronchoscopy: a randomized exploratory study of diagnostic value compared to standard white light bronchoscopy and autofluorescence bronchoscopy. Respir Res 16:33-37

33. Douplik A, Leong WL, Easson AM, Done S, Netchev G, Wilson BC (2009) Feasibility study of autofluorescence mammary ductoscopy. J Biomed Opt 14(4):44036

34. Waaijer L, Filipe MD, Simons J, van der Pol CC, de Boorder T, van Diest PJ et al (2020) Detection of breast cancer precursor lesions by autofluorescence ductoscopy. Breast Cancer 28:119

35. Lee GI, Lee MR, Green I, Allaf M, Marohn MR (2017) Surgeons' physical discomfort and symptoms during robotic surgery: a comprehensive ergonomic survey study. Surg Endosc 31(4):1697-1706 
36. Angarita FA, Castelo M, Englesakis M, McCready DR, Cil TD (2020) Robot-assisted nipple-sparing mastectomy: systematic review. Br J Surg 107(12):1580-1594

37. Zhang L, Jin K, Wang X, Yang Z, Wang J, Ma J et al (2019) The impact of radiotherapy on reoperation rates in patients undergoing mastectomy and breast reconstruction. Ann Surg Oncol 26(4):961-968
Publisher's Note Springer Nature remains neutral with regard to jurisdictional claims in published maps and institutional affiliations. 